\title{
Economic evaluation of the use of PCR assay in diagnosing pulmonary TB in a low-incidence area
}

\author{
I. Rajalahti*, E-L. Ruokonen", T. Kotomäki", H. Sintonen ", M.M. Nieminen*,+
}

Economic evaluation of the use of PCR assay in diagnosing pulmonary TB in a low-incidence area. I. Rajalahti, E-L. Ruokonen, T. Kotomäki, H. Sintonen, M.M. Nieminen. C) ERS Journals Ltd 2004.

ABSTRACT: To determine whether polymerase chain reaction (PCR) testing in the initial diagnosis of pulmonary tuberculosis (TB) is cost-effective in a low-prevalence population, an economic evaluation was carried out between the smear and culture (NOPCR) and smear, culture and PCR (+PCR) strategies.

A decision tree model based on retrospective laboratory data was developed to assess the strategies of testing patients with suspicion of TB. Direct healthcare costs prior to confirmation of TB or nontuberculous mycobacteria by PCR or culture were included. Effectiveness was measured by the probability of correct treatment and isolation decisions.

In the baseline situation NOPCR costs $€ 29.50$ less than the +PCR strategy per patient tested. According to sensitivity analyses, reducing PCR test price, shortening test performance time or increasing the proportion of smear-positive patients in the tested population would contribute to cost savings with the +PCR strategy.

Routine polymerase chain reaction testing of all specimens from suspected tuberculosis patients in a low-prevalence population was not cost-saving. When the polymerase chain reaction assay was applied only to smear-positive sputum specimens, the smear and culture strategy was clearly dominated by it, i.e. the polymerase chain reaction smear-positive sputum strategy was less costly and more effective in producing correct treatment decisions and isolations.

Eur Respir J 2004; 23: 446-451.
*Dept of Pulmonary Diseases, Tampere University Hospital, and ${ }^{+}$Dept of Respiratory Medicine, Medical School, University of Tampere, Tampere, " Dept of Pulmonary Diseases, Turku University Central Hospital, Preitilä, and Dept of Public Health, University of Helsinki, Helsinki, Finland.

Correspondence: I. Rajalahti, Dept of Pulmonary Diseases, Tampere University Hospital, PO Box 2000, FIN-33521 Tampere, Finland.

Fax: 35836292192

E-mail: iiris.rajalahti@kolumbus.fi

Keywords: Cost-effectiveness, diagnosis, polymerase chain reaction test, pulmonary tuberculosis

Received: January 282003

Accepted after revision: October 132003

This study was supported by the Finnish Anti-Tuberculosis Association Foundation, the Tampere Tuberculosis Foundation, the Research Fund of Tampere University, the Medical Research Fund of Tampere University Hospital, and Roche Ltd.
To date the global tuberculosis (TB) epidemic has shown no notable decline. In low-prevalence countries, factors contributing to the spread of the disease, especially in hospital settings, are delays in diagnosis and initiation of treatment [1]. In addition to lack of suspicion of TB, diagnosis is prolonged by the time-consuming identification of the Mycobacterium tuberculosis complex in sputum specimens [2]. The acid-fast smear rapidly identifies patients with infectious $\mathrm{TB}$, but is neither sensitive nor specific for $M$. tuberculosis bacteria. In countries like Finland (494 TB cases and 505 cases with nontuberculous mycobacteria in 2001) where nontuberculous mycobacteria are frequently detected, smear-positive nontuberculous cases may be isolated and treated with inappropriate drug combinations unnecessarily until culture results are available. Culture is sensitive in detecting mycobacteria for species identification and susceptibility testing, but requires a mean time of 2-3 weeks [3]. In smear-negative TB cases this may lead to unnecessary diagnostic procedures that increase healthcare costs and cause distress to the patients.

Commercial nucleic acid amplification (NAA) assays are rapid, sensitive and specific tools for the detection of the M. tuberculosis complex in sputum specimens [4-6]. In addition to rapid differentiation between this complex and nontuberculous mycobacteria in smear-positive sputum specimens, NAA assays detect a considerable proportion of smear-negative culture-positive TB cases [4, 6, 7]. However, the implementation and performance of these tests demands financial resources and experienced laboratory personnel; therefore the cost-effectiveness of NAA assays in diagnosing pulmonary TB should be carefully assessed.

Finland is classified as a low-prevalence country with the TB incidence below 10 per 100,000 inhabitants since 2001. In order to make an economic evaluation of two different strategies (smear, culture and polymerase chain reaction (PCR) testing to all specimens (+PCR), and smear and culture testing to all specimens, no PCR testing (NOPCR)) in diagnosing pulmonary TB in a low-prevalence population, a decision tree model was developed. For cost-minimisation analysis, all direct healthcare costs were included, prior confirmation or exclusion of $\mathrm{TB}$ or nontuberculous mycobacteria by culture or PCR. Effectiveness was measured by the probability of correct treatment and isolation decisions.

\section{Materials and methods}

A decision tree was developed to compare the expected costs and outcomes of two strategies for diagnosing pulmonary TB: the conventional NOPCR strategy currently used, based on smear and culture tests, and the +PCR strategy, in 
which a PCR test is performed on sputum specimens in addition to smear and culture. The model shows the paths from initial suspicion of TB to eventual outcomes with their associated probabilities and costs (fig. 1). In this analysis, the tree was used to calculate the expected cost per patient and the probability of correct treatment and isolation decisions for each strategy. The decision-making in the model proceeds according to the available test results and the patient's clinical picture. The baseline probabilities used in the decision tree were based on retrospective patient data from the 2-yr period 1997-1998. To level off yearly fluctuations in low-prevalence areas, the combined annual data of two large Finnish University Hospital Districts (Pirkanmaa and VarsinaisSuomi, populations of 446,000 and 449,000 inhabitants, respectively) were applied. The description of the branches and calculations of the baseline probabilities are presented in table 1 .

The baseline performance values for the PCR test were obtained from the study performed earlier in the Pirkanmaa Hospital District [6]. In that study 367 sputum specimens from 169 patients were tested by auramine fluorescent and Ziehl-Neelsen stain, Löwenstein-Jensen and radiometric liquid culture system cultures (BACTEC; Becton Dickinson Diagnostic Instrument Systems, MD, USA) and by the Cobas Amplicor PCR method (Roche Diagnostics, Basel, Switzerland).

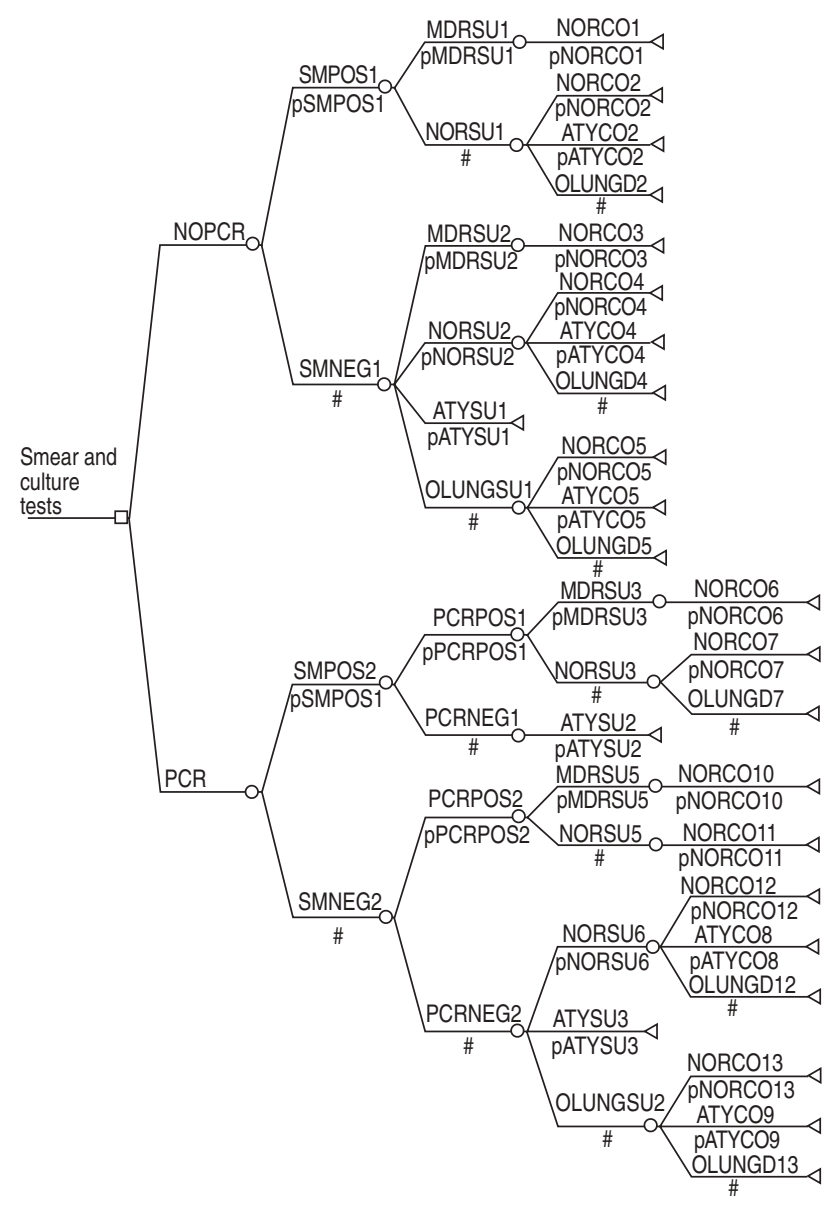

Fig. 1.-Decision tree showing paths to eventual outcomes in the smear and culture (NOPCR) and smear, culture and polymerase chain reaction $(+\mathrm{PCR})$ strategies. The prefix $(\mathrm{p})$ indicates "probability", numbers $(1,2,3$, etc.) are variable identifiers and residual probability is shown (\#; 1-the complementary probability). OLUNGD: other lung disease; OLUNGSU: other lung disease suspected; PCRNEG: PCR negative; SMNEG: smear negative. For other abbreviations see table 1 .
Positive cultures were identified by AccuProbe ribonucleic acid-deoxyribonucleic acid hybridisation (Gen-Probe Inc., San Diego, CA, USA). The sensitivities of the PCR test for smear-positive and smear-negative TB cases (three sputums per patient) were $100 \%$ and $75 \%$, respectively [6].

All subjects referred from primary healthcare and hospitals for sputum specimen examination due to suspicion of TB were included in the study population. According to the Finnish guidelines, pulmonary TB is suspected in a patient with prolonged cough and sputum expectoration, and/or infiltrations in chest radiograph. Additional criteria for sputum testing are unexplained weight loss, fatigue, night-sweating and/or prolonged fever. Multidrug-resistant (MDR) TB is suspected if the patient is a close contact of an MDR case or has stayed longer in a high-incidence area of MDR TB, whereas earlier detection of nontuberculous mycobacteria and/or a patient's chronic pulmonary disease support the suspicion of patient harbouring nontuberculous mycobacteria.

To clarify the decision tree model, the following baseline assumptions were made. For smear- and PCR-negative patients the decision on suspected disease (pulmonary TB, infection with nontuberculous mycobacteria, other disease) in the model was made on the basis of patient history and clinical picture. The accuracy in pulmonary clinicians suspecting the disease eventually verified was estimated to be $70 \%$. Further investigations or treatment were performed according to the suspected disease. These included high-resolution computed tomography of chest, bronchoscopy and treatment of suspected respiratory infection with antibiotics (levofloxacin for 7 days). Tuberculin testing was not included in view of its limited value in evaluating TB infection due to high coverage of BCG vaccination in Finnish population [8]. According to the Finnish guidelines, treatment regimen of the intensive phase was isoniazid, rifampin and pyrazinamid for the first 2 months. Ethambutol and streptomycin were added to the initial regimen if MDR TB was suspected. The isolation room conditions were similar for drug-sensitive and MDR TB patients. Patients suspected of harbouring nontuberculous mycobacteria in their lungs were not treated until culture results were available. Moreover, most such patients do not have a disease requiring treatment. All TB cases and cases with nontuberculous mycobacteria were confirmed by culture (combination of mycobacteria growth indicator tube and solid media). The average time for positive culture was estimated to be 2 weeks based on earlier studies [3,9] and the actual laboratory performance data. Susceptibility testing was completed in 8 weeks. The average growth rate of nontuberculous mycobacteria was assumed to be similar to that of $M$. tuberculosis. PCR tests were performed twice a week; thus PCR results were available to clinicians in 4 days, at the latest.

All use of healthcare resources prior to confirmation of correct diagnosis by culture or PCR was included in the evaluation and valued at the unit costs prevailing in the two hospital districts in 2000. The unit costs are presented in table 2. Since items such as contact tracing or patient costs (work time lost, transportation, distress from unnecessary isolation and diagnostic procedures) are difficult to establish and may vary considerably individually, these were not estimated in this study.

The robustness of the findings to changes in the main parameters in the model was tested by three types of sensitivity analysis. Firstly, the sensitivity was tested in terms of time when the PCR and culture results were available (table 3). Secondly, threshold analyses were performed to find out the values of some key variables with which the expected cost/patient is equal in the +PCR and NOPCR alternatives (table 4). Thirdly, two-way sensitivity analysis was carried out to see the possible combinations of 
Table 1.-Abbreviations, descriptions and baseline probabilities used in decision tree analysis

\begin{tabular}{|c|c|c|c|c|c|}
\hline \multirow[t]{2}{*}{ Branch } & \multirow[t]{2}{*}{ Description } & \multirow[t]{2}{*}{$\mathrm{n}$} & \multicolumn{3}{|c|}{ Probability } \\
\hline & & & Variable & Description & Value \\
\hline SMPOS1 & Smear positive & 38 & pSMPOS1 & $\mathrm{SMPOS}^{\#}{ }^{\#} \mathrm{~N}^{\bullet}$ & 0.031 \\
\hline MDRSU1 & Multidrug-resistant TB suspected & 3 & pMDRSU1 & MDRSU1§/SMPOS1 & 0.079 \\
\hline MDRSU2 & Multidrug-resistant TB suspected & 1 & pMDRSU2 & MDRSU2$^{\S} / \mathrm{SMNEG}^{+}$ & 0.001 \\
\hline MDRSU3 & Multidrug-resistant TB suspected & 3 & pMDRSU3 & MDRSU3/PCRPOS1 & 0.150 \\
\hline MDRSU5 & Multidrug-resistant TB suspected & 1 & pMDRSU5 & MDRSU5 $5 /$ PCRPOS2 & 0.167 \\
\hline NORSU2 & Drug-sensitive TB suspected & 10 & pNORSU2 & NORSU2/SMNEG1 & 0.008 \\
\hline NORSU6 & Drug-sensitive TB suspected & 3 & pNORSU6 & NORSU6 ${ }^{\S} / \mathrm{PCRNEG} 2$ & 0.003 \\
\hline ATYSU1 & Nontuberculous mycobacteria suspected & 21 & pATYSU1 & ATYSU1§/SMNEG1 & 0.018 \\
\hline ATYSU2 & Nontuberculous mycobacteria suspected & 18 & pATYSU2 & ATYSU2/PCRNEG1 & 1.000 \\
\hline ATYSU3 & Nontuberculous mycobacteria suspected & 21 & pATYSU3 & ATYSU3\$/PCRNEG2 & 0.018 \\
\hline PCRPOS1 & PCR positive & 20 & pPCRPOS1 & PCRPOS1/SMPOS2 ${ }^{\#}$ & 0.526 \\
\hline PCRPOS2 & PCR positive & 6 & pPCRPOS2 & PCRPOS2/SMNEG2 $^{+}$ & 0.005 \\
\hline NORCO1 & Drug-sensitive TB confirmed & 3 & pNORCO1 & NORCO1/MDRSU1 & 1.000 \\
\hline $\mathrm{NORCO} 2$ & Drug-sensitive TB confirmed & 16 & pNORCO2 & NORCO2/NORSU1 & 0.457 \\
\hline NORCO3 & Drug-sensitive TB confirmed & 1 & pNORCO3 & NORCO3/MDRSU2 & 1.000 \\
\hline NORCO4 & Drug-sensitive TB confirmed & 5 & pNORCO4 & NORCO4/NORSU2 & 0.500 \\
\hline NORCO5 & Drug-sensitive TB confirmed & 1 & pNORCO5 & NORCO5/OLUNGSU1 & 0.001 \\
\hline NORCO6 & Drug-sensitive TB confirmed & 3 & pNORCO6 & NORCO6/MDRSU3 & 1.000 \\
\hline NORCO7 & Drug-sensitive TB confirmed & 16 & pNORCO7 & NORCO7/NORSU3 & 0.941 \\
\hline NORCO10 & Drug-sensitive TB confirmed & 1 & pNORCO10 & NORCO10/MDRSU5 & 1.000 \\
\hline NORCO11 & Drug-sensitive TB confirmed & 5 & pNORCO11 & NORCO11/NORSU5 & 1.000 \\
\hline NORCO12 & Drug-sensitive TB confirmed & 1 & pNORCO12 & NORCO12/NORSU6 & 0.333 \\
\hline NORCO13 & Drug-sensitive TB confirmed & 1 & pNORCO13 & NORCO13/OLUNGSU2 & 0.001 \\
\hline ATYCO2 & Nontuberculous mycobacteria confirmed & 15 & pATYCO2 & ATYCO2/NORSU1 & 0.429 \\
\hline ATYCO4 & Nontuberculous mycobacteria confirmed & 4 & pATYCO4 & ATYCO4/NORSU2 & 0.400 \\
\hline ATYCO5 & Nontuberculous mycobacteria confirmed & 2 & pATYCO5 & ATYCO5/OLUNGSU1 & 0.002 \\
\hline ATYCO8 & Nontuberculous mycobacteria confirmed & 1 & pATYCO8 & ATYCO8/NORSU6 & 0.333 \\
\hline ATYCO9 & Nontuberculous mycobacteria confirmed & 5 & pATYCO9 & ATYCO9/OLUNGSU2 & 0.004 \\
\hline
\end{tabular}

${ }^{\#}: \mathrm{n}=38$, including 19 patients with tuberculosis (TB), 15 with nontuberculous mycobacteria and four with negative cultures; ${ }^{\top}$ : baseline population, $\mathrm{n}=1,219 ;^{+}: \mathrm{n}=1,181$, including eight patients with TB and 20 patients with nontuberculous mycobacteria; ${ }^{\S}$ : based on expert opinion.

Table 2. - Unit costs used in decision tree analysis

\begin{tabular}{lr}
\hline Cost items & $€$ \\
\hline TB isolation 14 days & 4768.10 \\
TB medication per week & 26.70 \\
Laboratory tests, isolation & 20.70 \\
Multidrug-resistant TB isolation 14 days & 4768.10 \\
Multidrug-resistant TB medication per month & 165.80 \\
Inpatient care per day & 169.00 \\
Outpatient visit & 64.70 \\
Smear and culture $\times 3$ & 70.60 \\
PCR test $\times 3$ & 126.10 \\
Chest radiograph & 27.20 \\
Laboratory tests, control & 17.70 \\
High resolution computed tomography & 154.10 \\
Bronchoscopy & 235.10 \\
Medication against infection & 27.80
\end{tabular}

TB: tuberculosis; PCR: polymerase chain reaction.

the price of the PCR test and the probability of smear-positive patients, with which the +PCR strategy may be cost-saving (fig. 2).

\section{Results}

The baseline population in the decision tree model was 1,219 patients, including $27(2.2 \%)$ culture-proven pulmonary TB patients and $35(2.8 \%)$ patients with nontuberculous mycobacteria. No MDR TB patients were detected in the study districts during 1997-1998, while two MDR cases were reported overall in Finland during that period. The proportion of smear-positive patients was $3.1 \%$. Of the 38 smear-positive patients, 19 were TB patients and 15 patients had nontuberculous mycobacteria. Furthermore, four patients proved positive by smear but negative by culture, most probably due to slowly growing or unculturable nontuberculous mycobacterial species.

The results of the base case situation and sensitivity analyses are presented in tables 3 and 4 . In the base case, where PCR results were available in 4 days and culture results in 2 weeks, the cost per patient tested in the NOPCR strategy was $€ 29.50(12 \%)$ less than in the +PCR strategy (table 3 ). Prolonging culturing time to 3 weeks did not change this result. In the ideal setting for PCR, in which PCR is performed daily and the culturing time is 3 weeks, the + PCR strategy would save $€ 8.90$ per patient tested compared with the NOPCR strategy (table 3 ).

The model proved to be sensitive to the proportion of smear-positive patients in the base case population and to the cost of the PCR test. With baseline assumptions the threshold analysis showed that if the proportion of smear-positive patients in the tested population were over $4.0 \%$, the PCR strategy would be cost-saving. Similarly, if the cost of the PCR test (including a set of three sputum tests) was $\leqslant € 97$, the +PCR strategy would reduce costs compared with NOPCR. Additionally, threshold analysis revealed that if isolation expenses rose above $€ 5,965$ per case isolated in the base situation, the +PCR strategy would be less costly. The model was robust to changes both in the probability of smearpositive PCR-positive cases (pPCRPOS1) and smear-negative PCR-positive cases (pPCRPOS2) and no threshold values in reasonable ranges were found (table 4). Furthermore, twoway sensitivity analysis indicates that the less expensive PCR 
Table 3. - The results of base case and sensitivity analyses

\begin{tabular}{lccc}
\hline Analysis & Strategy & Cost/patient tested $€$ & Incremental cost $€$ \\
\hline Base analysis & NOPCR & 225.40 \\
PCR results in 4 days, culture results in 2 weeks & + PCR & 254.90 & 226.40 \\
Sensitivity analysis 1 & NOPCR & 254.90 & 29.50 \\
PCR results in 4 days, culture results in 3 weeks & + PCR & 226.40 & 28.50 \\
Sensitivity analysis 2 & NOPCR & 217.50 & -8.90 \\
PCR results in 1 day, culture results in 3 weeks & +PCR & 225.40 & 130.00 \\
Sensitivity analysis 3 & NOPCR & -95.40 \\
PCR results in 4 days, culture results in 2 weeks & +PCR SMPOS & \\
\hline
\end{tabular}

NOPCR: smear and culture testing to all specimens, no polymerase chain reaction (PCR) testing; + PCR: smear, culture and PCR testing to all specimens; +PCR SMPOS: smear and culture testing to all specimens, and PCR testing only to smear-positive specimens.

Table 4. - The variables selected for threshold analysis and its results (base case)

\begin{tabular}{|c|c|c|c|c|}
\hline Variable & Range & TV & $\begin{array}{l}\text { Optimal strategy, if } \\
\text { variable value }<\mathrm{TV}\end{array}$ & $\begin{array}{l}\text { Optimal strategy, if } \\
\text { variable value }>T V\end{array}$ \\
\hline \multicolumn{5}{|l|}{ Probabilities } \\
\hline pSMPOS1 & $0.01-0.08$ & 0.04 & NOPCR & $+\mathrm{PCR}$ \\
\hline pPCRPOS1 & $0.35-0.70$ & No threshold & & \\
\hline pPCRPOS2 & $0.001-0.09$ & No threshold & & \\
\hline \multicolumn{5}{|l|}{ Costs $€$} \\
\hline $\mathrm{PCR}^{\#}$ & $50-200$ & 96.60 & $+\mathrm{PCR}$ & NOPCR \\
\hline TBISO & $4500-6000$ & 5964.60 & NOPCR & +PCR \\
\hline MDRISO & $4500-6000$ & No threshold & & \\
\hline
\end{tabular}

TV: threshold value; pSMPOS1: probability of smear-positive patients; pPCRPOS1: probability of smear and polymerase chain reaction (PCR)positive patients; pPCRPOS2: probability of smear-negative, PCR-positive patients; TBISO: isolation of drug-sensitive tuberculosis (TB) patient for 14 days; MDRISO: isolation of multidrug-resistant TB patient for 14 days. ${ }^{\#}$ : set of three PCR tests per patient.

testing is, the smaller the proportion of smear-positive patients in the tested population can be for the +PCR strategy to be cost-saving (fig. 2). The threshold value for the cost of a PCR test (a set of three tests) can be determined from figure 2 when the incidence of smear-positive patients in the population is known.

The effectiveness of the +PCR and NOPCR strategies in terms of correct treatment and isolation decisions was also evaluated. In the base case situation the incremental cost turned out to be $€ 1,970$ for one additional correct treatment decision and $€ 2,011$ for an additional correct isolation with the +PCR strategy.

The alternative strategy of applying PCR testing only to smear-positive sputum specimens (+PCR SMPOS strategy) proved to be cost-effective and dominated the NOPCR strategy, which means that in addition to being clearly less costly (€95.30 per patient cheaper), more correct treatment decisions were obtained (1.47 percentage points difference in favour of +PCR SMPOS strategy) and almost one-half of isolations were avoided with the +PCR SMPOS strategy (table 5).

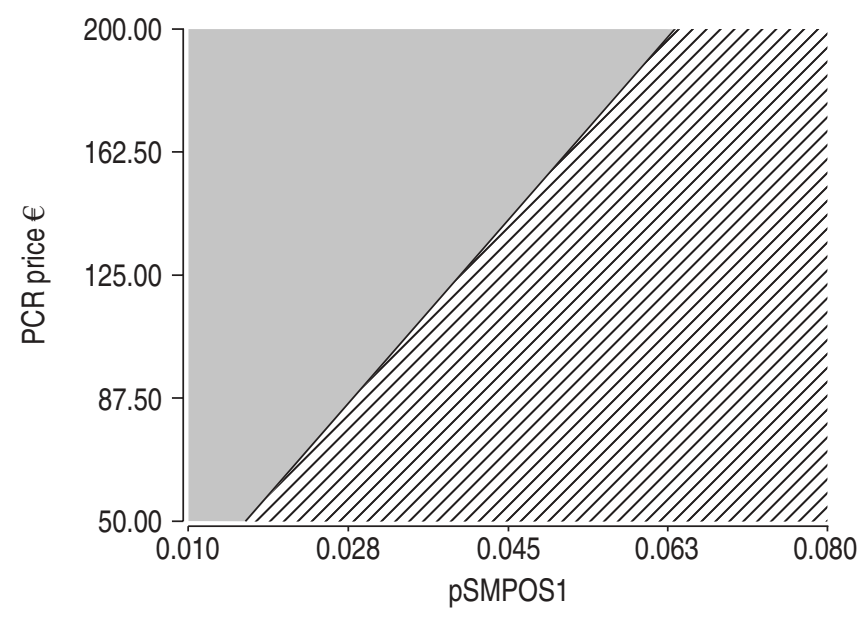

Fig. 2. - Two-way sensitivity analysis with polymerase chain reaction (PCR) price and proportion of smear-positive patients in the population (pSMPOS1). : NOACR strategy is cost-saving; $\mathbb{Z}:+\mathrm{PCR}$ strategy is cost-saving.

Table 5. - Cost-effectiveness analysis of correct treatment and isolation decisions

\begin{tabular}{|c|c|c|c|c|c|c|}
\hline Strategy & $\begin{array}{c}\text { Cost per patient } \\
\text { tested } €\end{array}$ & $\begin{array}{l}\text { Incremental } \\
\text { cost } €\end{array}$ & Effectiveness ${ }^{\#}$ & $\begin{array}{l}\text { Incremental } \\
\text { effectiveness }\end{array}$ & $\begin{array}{c}\text { Cost-effectiveness } \\
\text { ratio } €\end{array}$ & $\begin{array}{l}\text { Incremental cost- } \\
\text { effectiveness ratio }\end{array}$ \\
\hline \multicolumn{7}{|c|}{ Correct treatment decisions } \\
\hline +PCR SMPOS & 130.00 & & 0.9716 & & 133.84 & \\
\hline NOPCR & 225.40 & 95.30 & 0.9569 & -0.0147 & 235.52 & Dominated \\
\hline \multicolumn{7}{|c|}{ Correct isolation decisions } \\
\hline +PCR SMPOS & 130.00 & & 0.0302 & & 4308.55 & \\
\hline NOPCR & 225.40 & 95.30 & 0.0155 & -0.0147 & 14543.73 & Dominated \\
\hline
\end{tabular}

+PCR SMPOS: smear and culture testing to all specimens, and PCR testing only to smear-positive specimens; NOPCR: smear and culture testing to all specimens, no PCR testing. ${ }^{\#}$ : probability of correct decision. 


\section{Discussion}

Rapid confirmation of TB diagnosis is an essential part of the TB control strategy. Features such as standardised reagents, automated reading of reaction results, detection of inhibition and rapidity of the test make Amplicor PCR suitable for a routine screening test for $M$. tuberculosis detection [10]. It cannot, however, replace the smear, which determines infectiousness, or culture, which is essential in species identification and susceptibility testing. Furthermore, combined use of these three tests is limited mainly by the cost of PCR testing, and therefore, economic evaluation is needed to assess the role of PCR testing.

According to the present data the NOPCR strategy was cost-saving compared with +PCR when the PCR assay was applied to all specimens from suspected TB patients and performed twice a week. The sensitivity analyses indicated three significant factors contributing to the costs of the +PCR strategy: the performance time and the cost of the PCR assay, as well as the proportion of smear-positive patients in the base population.

Performing the PCR assay twice a week is a realistic frequency in a low-prevalence area. More frequent test runs would demand larger numbers of specimens, whereas testing once a week would compromise the rapidity of the PCR assay, which is its most important feature. The current PCR test expenses originate predominately from expensive reagents. Reducing the number of sputum specimens tested per patient from three to two would reasonably decrease the costs. However, multiple specimens are required to maximise the sensitivity of the PCR test in the detection of M. tuberculosis especially in smear-negative specimens, and to confirm the exclusion of $M$. tuberculosis in smear-positive specimens [6, 7, 10]. The fact that patients expectorate bacteria infrequently and specimens are of inconsistent quality is supported by the findings of NELSON et al. [11] in which 13\% of smear-positive and $7 \%$ of smear-negative TB cases were detected by conventional tests only from the third sputum specimen tested.

According to two-way sensitivity analysis, the expenses of the PCR strategy could be reduced by controlling the proportion of smear-positive patients in the population tested. In this model, only $3.1 \%$ of patients were positive by smear and $2.1 \%$ had pulmonary TB confirmed by culture, reflecting an unnecessarily low threshold among clinicians in suspecting pulmonary TB. It may be explained by scanty clinical experience of $\mathrm{TB}$ in public healthcare facilities, whereas in hospital settings sputum examination for $M$. tuberculosis may be performed routinely for most patients with respiratory symptoms. In a study by DIVINAGRACIA et al. [12], sputum testing by smear and culture was indicated in only $\sim 53 \%$ of all suspected TB cases; nevertheless no TB cases were missed. Further, a clinical risk assessment for TB suspicion proved to help targeting patient populations who would benefit from PCR testing [13]. More accurate selection of patient population would reduce unnecessary testing of patients with other diseases. However, in a low-prevalence population the total number of submitted specimens would decrease to a level where performing PCR tests would be appropriate only once a week. This may be avoided by centralising specimen testing in one laboratory centre, but consequent transportation, specimen handling and result service are matters still to be resolved and were not evaluated in this study.

The results of the analysis were robust to changes in the probability of smear-positive PCR-positive cases (pPCRPOS1), which reflects the sensitivity of the PCR test for smearpositive TB cases (table 4). This is explained by those smear-positive TB patients misdiagnosed as patients with nontuberculous mycobacteria who would incur no additional costs in the model prior to culture confirmation. However, epidemiologically these infectious smear-positive TB patients would have a notable impact on TB control. The threshold value for pPCRPOS2, expressing the sensitivity of the PCR test for smear-negative TB cases, was not detected due to the small proportion of smear-negative TB patients in the population tested. A clinician's likelihood to suspect the correct diagnosis with an accuracy of $70 \%$ in smear-negative patients may have been overestimated in the model. However, sensitivity analysis revealed that the economic benefit of the NOPCR strategy remained unchanged even when the accuracy of suspicion was only $50 \%$. This is also explained by the small proportion $(0.7 \%)$ of smear-negative TB patients in the population, and hence its minimal effect on the total costs of the laboratory strategies.

In the baseline situation PCR testing of all suspected TB patients was not profitable in a low prevalence country. It cost $€ 29.50$ more per patient tested and the cost for one additional correct treatment decision was $€ 1,970$. General assessment of acceptable incremental cost for applying PCR strategy was not feasible in this study. It is highly dependent on the local TB infection control strategy and fiscal framework, and has to be evaluated separately in each setting concerned.

The highest expenses per patient in both strategies originated from isolation and treatment of smear-positive patients. The average cost per smear-positive patient was $€ 5,006$ in the NOPCR strategy and $€ 1,931$ in $+P C R$. Isolation room costs may constitute up to $87 \%$ of total costs in the treatment of smear-positive TB patients [14]. On these grounds, application of the PCR assay only to smear-positive specimens would be reasonable. The present findings indicate that the +PCR smear-positive strategy is clearly cost-effective compared with the NOPCR strategy. Rapid differentiation between $M$. tuberculosis and nontuberculous mycobacteria by PCR results in infection control cost-savings by reducing inpatient care days and unnecessary isolations. Furthermore, needless contact tracing and patient distress are avoided, which were not measured in this study. However, as mentioned, application of the +PCR smear-positive strategy would require centralised specimen testing.

The absence of MDR cases in the model did not influence the cost-effectiveness evaluation because the PCR assay does not differentiate between drug-sensitive and MDR TB. Costs of treating MDR patients or suspects were thereby equal, regardless of the laboratory strategy chosen. Molecular rifampin resistance testing was not included in this model.

Costs included in the decision tree were those prevailing in Finnish healthcare. These and clinical practice patterns used in the model may vary between countries. The cost of the PCR kit may differ depending on the supplier, and the use of homemade kits and the sensitivity of the test applied is influenced by the extraction, amplification and final interpretation methods of the results used. Finally, a notable factor contributing interpretation of the results is the incidence of nontuberculous mycobacteria and its portion of the smear-positive patient population.

According to the present results, routine application of polymerase chain reaction testing to all specimens from suspected tuberculosis patients in a low-prevalence setting is not cost-saving. However, reduction of polymerase chain reaction assay expenses and more selective screening of the patient population would contribute to cost reductions compared with the no polymerase chain reaction strategy. If the polymerase chain reaction assay were applied only to smear-positive sputum specimens, the polymerase chain reaction smear-positive strategy would be the dominant strategy, that is, less costly and more effective in leading to correct treatment decisions and isolations. Polymerase 
chain reaction testing would be particularly beneficial in populations with a high prevalence of nontuberculous mycobacteria.

\section{References}

1. Rao VK, Iademarco EP, Fraser VJ, Kollef MH. Delays in the suspicion and treatment of tuberculosis among hospitalized patients. Ann Intern Med 1999; 130: 404-411.

2. Mathur P, Sacks L, Auten G, Sall R, Levy C, Gordin F. Delayed diagnosis of pulmonary tuberculosis in city hospitals. Arch Intern Med 1994; 154: 306-310.

3. Pfyffer GE, Welscher H-M, Kissling P, et al. Comparison of the mycobacteria growth indicator tube (MGIT) with radiometric and solid culture for recovery of acid-fast bacilli. J Clin Microbiol 1997; 35: 364-368.

4. Gamboa F, Fernandez G, Padilla E, et al. Comparative evaluation of initial and new versions of the Gen-Probe amplified Mycobacterium tuberculosis direct test for direct detection of Mycobacterium tuberculosis in respiratory and nonrespiratory specimens. J Clin Microbiol 1998; 36: 684-689.

5. Ichiyama S, Iinuma $\mathrm{Y}$, Tawada $\mathrm{Y}$, et al. Evaluation of Gen-Probe amplified Mycobacterium tuberculosis direct test and Roche PCR-microwell plate hybridization method (AMPLICOR MYCOBACTERIUM) for direct detection of mycobacteria. J Clin Microbiol 1996; 34: 130-133.

6. Rajalahti I, Vuorinen P, Nieminen MM, Miettinen A. Detection of Mycobacterium tuberculosis complex in sputum specimens by the Automated Roche Cobas Amplicor Mycobacterium Tuberculosis test. J Clin Microbiol 1998; 36: 975-978.

7. Della-Latta $\mathrm{P}$, Whittier S. Comprehensive evaluation of performance, laboratory application, and clinical usefulness of two direct amplification technologies for the detection of Mycobacterium tuberculosis complex. Am J Clin Pathol 1998; 110: 301-310.

8. Härö AS. Long-term evaluation of mass BCG-vaccination campaign: a study of 30 years of experience in Finland. In: Tuberculosis and Respiratory Diseases. Helsinki, Year book of Finnish Anti-tuberculosis Association, 1977; p. 6.

9. Flanagan PG, Williams R, Paull A. Comparison of two automated systems for the isolation of mycobacteria from clinical specimens. Eur J Clin Microbiol Infect Dis 1999; 18: 912-914.

10. Scarparo C, Piccoli P, Rigon A, Ruggiero G, Scagnelli M, Piersimoni C. Comparison of enhanced Mycobacterium tuberculosis amplified direct test with COBAS AMPLICOR Mycobacterium tuberculosis assay for direct detection of Mycobacterium tuberculosis complex in respiratory and extrapulmonary specimens. J Clin Microbiol 2000; 38: $1559-1562$.

11. Nelson SM, Deike MA, Cartwright CP. Value of examining multiple specimens in the diagnosis of pulmonary tuberculosis. J Clin Microbiol 1998; 36: 467-469.

12. Divinagracia RM, Harkin TJ, Bonk S, Schluger NW. Screening by specialists to reduce unnecessary test ordering in patients evaluated for tuberculosis. Chest 1998; 114: 681684.

13. Catanzaro A, Perry S, Clarridge JE, et al. The role of clinical suspicion in evaluating a new diagnostic test for active tuberculosis. JAMA 2000; 283: 639-645.

14. Wurtz R, White WD. The cost of tuberculosis: utilization and estimated charges for the diagnosis and treatment of tuberculosis in a public health system. Int J Tuberc Lung Dis 1999; 3: 382-387. 